\title{
A secondary analysis of atypical presentations of older patients with infection in the emergency department of a tertiary care hospital in Thailand
}

\author{
Panita Limpawattana ${ }^{\mathrm{a}}$, Thapanawong Mitsungnern ${ }^{\mathrm{b}}$, Pariwat Phungoen ${ }^{\mathrm{b}}$, Natthida Tansangwornc, \\ Wannisa Laosuangkoon ${ }^{\mathrm{C}}$ \\ ${ }^{a}$ Division of Geriatric Medicine, Department of Internal Medicine, Faculty of Medicine, Khon Kaen \\ University, Khon Kaen 40002, Thailand \\ ${ }^{b}$ Department of Emergency Medicine, Faculty of Medicine, Khon Kaen University, Khon Kaen 40002, \\ Thailand \\ 'Department of Internal Medicine, Faculty of Medicine, Khon Kaen University, Khon Kaen 40002, \\ Thailand
}

\begin{abstract}
Background: Classic symptoms of illness are often absent in older adults, delaying diagnosis and management, and resulting in unfavorable outcomes.

Objectives: To determine the prevalence of atypical presentations by older Thai adults with infections in the emergency department (ED) of a tertiary care hospital, and to identify factors associated with atypical presentations in such patients.

Methods: We performed a secondary analysis of a retrospective cohort observational study of atypical presentations of patients aged $\geq 65$ years in the ED using a subset of data first reported in the Archives of Gerontology and Geriatrics in 2015; 62:97-102.

Results: The prevalence of atypical presentations was 35\% of those with infectious diseases (64 of 183 cases). Pneumonia was the most common (30\%). We identified 4 associated risk factors: complicated urinary tract infection (UTI) (odds ratio (OR) 4.54; 95\% confidence interval (CI) 1.75,11. 78; $P=0.002$ ), cancer (OR 2.64; 95\% CI 1.07, 6.53; $P=0.04$ ), dementia (OR 6.66; 95\% CI 1.47, 30.11; $P=0.01$ ), and pulse rate $>90$ beats/min (OR 2.06; $95 \%$ CI 1.01, 4.22; $P=0.04$ ). Infective diarrhea was protective (OR 0.27; 95\% CI 0.09, 0.8; $P=0.02$ ).

Conclusions: Atypical presentations of infectious diseases by older Thai patients in the ED accounted for about one third of those with infectious disease. Associated risk factors were complicated UTI, cancer, dementia, and increased pulse rate. Older patients with these factors who have uncertain diagnoses should be considered for hospitalization because the absence of classic symptoms does not exclude life-threatening infections.
\end{abstract}

Keywords: Geriatrics, geriatric syndrome, infection presentation

Infectious diseases are frequent in older patients presenting to emergency departments (EDs). Existing studies show prevalence of infection ranging from $3 \%-14 \%$ [1-3]. Pneumonia is the leading cause in several reports, followed by urinary tract infection (UTI), and sepsis [3, 4]. Atypical presentations, including symptoms of specific diseases with absence of expected fever, and syndromes such as changes in consciousness, urinary incontinence, and falls

Correspondence to: Panita Limpawattana, Division of Geriatric Medicine, Department of Medicine, Faculty of Medicine, Khon Kaen University, Khon Kaen 40002, Thailand.

E-mail: lpanit@kku.ac.th are common in geriatric patients [4-6]. Atypical presentations were found in approximately $20 \%$ of elderly patients in the ED [7]. Only $20 \%-30 \%$ of geriatric patients with infectious diseases presented with fever at the ED. Therefore, looking for other factors that predict increased mortality related to infection is crucial. These factors include altered mental status, vomiting, tachypnea, tachycardia, and leukocytosis or elevated band form $>6 \%$ in the presence of normal white counts [5]. However, these factors may not be found in all elderly patients. They may have less tachycardia or absence of marked blood count modifications, and yet present with otherwise serious complications of infectious diseases including 
higher risks of developing acute respiratory failure, renal failure, and septic shock [8]. Only 20\% of older patients with acute appendicitis presented with classic pain and more than a half of these patients may have a blunted fever response [3]. Frequency of urination and burning or painful sensations during urination in patients who have urinary tract infection usually decrease with advancing age [9]. Patients are at risk of infection as a result of multimorbidity such as diabetes, chronic lung disease, malignancy, increased exposure to medical instrumentation and procedures, immunosenescence, malnutrition, poor performance status, and social and psychiatric factors $[4,5,10]$. These contributing factors lead to increases in morbidity and mortality, recurrent infection, organ failure, polypharmacy, and cognitive impairment. They cause increased healthcare costs and worsen functional status [2, 3, 7]. Patients with typical symptoms may have a protective factor leading to earlier assessment and prediction of higher-risk factors, and initiation of therapeutic intervention [11]. This study aimed to determine the prevalence of atypical presentations among older Thai patients with infectious diseases presenting at the ED of a tertiary care hospital and identification of factors associated with these presentations.

\section{Materials and methods Study participants}

This is secondary analysis of a retrospective cohort observational study of atypical presentations of older adults at an ED first reported in the Archives of Gerontology and Geriatrics in 2015 [12]. We analyzed a subset of data from patients diagnosed as having infectious diseases collected retrospectively in the previous study from the medical records of patients aged $\geq 65$ years who attended the ED of Srinagarind Medical School Hospital in 2013. Exclusion criteria were incomplete medical records. Only data from the first visit of patients with several visits during the studied period was analyzed [12]. Approval for the original study on which the present study is based was provided by the Ethics Committee for Human Research of Khon Kaen University following the guiding principles of the Declaration of Helsinki and IHC Good Clinical Practice (reference No. HE571004).

\section{Definitions}

Atypical presentation was defined as "a patient with no expected typical signs or symptoms expected for a final diagnosis or unusual signs and symptoms that are unrelated or even the opposite of what is usually expected including a vague presentation of the illness, altered clinical presentation of illness, and nonpresentation of illness, such as absence of dysuria, or frequent urination in patients with urinary tract infection, and a lack of fever, cough, or dyspnea in patients with lower respiratory tract infection. Specifically, we noted patients with septic shock who displayed no fever or tachycardia” [13, 14]. Multimorbidity was defined as "the co-occurrence of 2 or more medical or psychiatric conditions, which may or may not directly interact with each other within the same individual" [15].

The standardized format for recording diagnosis in this study was the International Classification of Diseases and Related Health Problems $10^{\text {th }}$ Revision (ICD-10). Diagnoses were based on clinical manifestations with or without laboratory investigations at the ED according to a standard medical textbook [16]. Infectious diseases were defined as illnesses "caused by a specific infectious agents, or their toxic products, that results from transmission of that agent or its products from an infected person, animal, or reservoir to a susceptible host either directly or indirectly through an intermediate plant or animal host, vector or inanimate object" [17].

Fever was identified when oral temperature was $37.8^{\circ} \mathrm{C}$ or over at the ED. This cut off provides lower sensitivity in detecting fever in older adults. Our study also identified a temperature of $37.2^{\circ} \mathrm{C}$ or over as fever if documented in prior reports [5].

\section{Procedure}

There were 10,438 patient visits to the ER. We selected 633 medical records using a linear systemic sampling method and 183 (28.9\%) patients were found to have infectious disease. The demographic data of the patients including age, sex, medical histories, current diagnoses, current numbers of medications, and initial vital signs at the ED were recorded by 5 physicians (with 2-15 years' experience in medical practice). Cases were discussed among the physicians to avoid potential bias.

\section{Sample size}

Sample-size calculations were based on the main objective of the present study, which was to estimate prevalence of atypical presentations in older adults with 
infectious disease at the $\operatorname{ED}[3,18]$. To calculate the estimated population proportion we used a specified absolute precision formula [19]. A sample size of at least 60 participants was sufficient to achieve this analysis at a level of significance of 0.05 .

\section{Statistical analyses}

Demographic data are presented using descriptive statistics, including percentages, means, and standard deviations. If the distribution of these data was not normal, medians, and interquartile ranges were used instead. The factors associated with the atypical presentation of infectious diseases were evaluated using univariate logistic regression. $P<0.05$ was considered to indicate significant differences between groups. Unadjusted odds ratios (OR) and their 95\% confidence intervals (CI) were reported to indicate the strength of association. For multiple logistic regression analyses, the numbers of variables entered into the multiple regression models was about 5-10 times of the number of risk factors [20]. After multicollinearity was checked, the selected variables which had $P<0.20$ from univariate analyses, or factors with clinical importance, were then entered into a multiple logistic regression model. $P<0.05$ was considered to indicate significant differences and adjusted odds ratios (OR) and their 95\% confidence intervals (CI) are reported to indicate the strength of association. All the data analyses were conducted using STATA, version 10.0 (Stata Corp, College Station, TX, USA).

\section{Results \\ Prevalence of atypical presentations}

We reviewed medical records of 633 older adults who visited the ED. Infectious diseases were found in 183 (28.9\%) cases, and 64 presented atypically according to the definition presented above. The demographic data of patients studied are displayed in Table 1. Common atypical sign or symptom presentations of common infectious diseases are shown in Figure 1.

\section{Factors associated with atypical presentations}

Comparison of the risk factors for atypical and typical presentations of older adults with infectious diseases at the ED using univariate analysis is shown in Table 1. For multivariate analysis, 7 factors where $P<0.2$ from the univariate analysis were entered into the multiple regression model: age, diagnosis of complicated UTI, infective diarrhea, skin infection, comorbid disease of dementia and cancer, and pulse rate $>90$ beats/min (Table 2). Complicated UTI, a background of dementia, cancer, and tachycardia were factors associated with atypical presentations in the present study. Multimorbidity is the co-occurrence of 2 or more medical or psychiatric conditions [15].

Table 1. Demographic data and univariate analysis of atypical and typical presentations of older adults with infectious diseases at the emergency department

\begin{tabular}{lllll}
\hline Factors & $\begin{array}{l}\text { Atypical } \\
(\mathbf{n = 6 4 )}\end{array}$ & $\begin{array}{l}\text { Typical } \\
(\mathbf{n = 1 1 9})\end{array}$ & $\begin{array}{c}\text { Unadjusted OR } \\
\mathbf{( 9 5 \% ( C I )}\end{array}$ & $\boldsymbol{P}$ \\
\hline Age (median, IQR 1, 3) & $75(71,80)$ & $73(68,78)$ & $1.05(1.0,1.09)$ & $0.04^{*}$ \\
Male (\%) & $31(47)$ & $35(53)$ & $1.06(0.58,1.94)$ & 0.86 \\
Top 5 diagnoses (\%) & & & \\
Acute pneumonia/ bronchitis & $20(36)$ & $35(64)$ & $1.11(0.57,2.13)$ & 0.76 \\
Infective diarrhea & $5(13)$ & $35(88)$ & $0.2(0.08,0.55)$ & $0.002^{*}$ \\
Complicated UTI & $20(69)$ & $9(31)$ & $5.56(2.36,13.1)$ & $<0.001^{*}$ \\
Skin infection & $3(83)$ & $15(17)$ & $0.1(0.06,0.17)$ & $<0.001^{*}$ \\
Septic shock & $7(47)$ & $8(53)$ & $1.72(0.6,5)$ & 0.32 \\
Comorbid (\%) & & & & \\
Hypertension & $35(37)$ & $59(63)$ & $1.23(0.67,2.26)$ & 0.51 \\
Diabetes mellitus & $14(28)$ & $37(73)$ & $0.62(0.31,1.26)$ & 0.19 \\
Musculoskeletal diseases & $11(33)$ & $22(67)$ & $0.92(0.41,2.03))$ & 0.83 \\
Dyslipidemia & $14(47)$ & $16(53)$ & $1.8(0.82,3.98)$ & 0.15 \\
Chronic kidney disease & $10(35)$ & $19(66)$ & $0.97(0.42,2.24)$ & 0.95 \\
Cancer & $15(54)$ & $13(46)$ & $2.5(1.1,5.64)$ & $0.03^{*}$ \\
Cerebrovascular accident & $8(69)$ & $18(31)$ & $0.8(0.33,1.96)$ & 0.63 \\
Coronary artery disease & $5(56)$ & $4(44)$ & $2.43(0.63,9.41)$ & 0.2 \\
Dementia & $7(70)$ & $3(30)$ & $4.75(1.18,19.05)$ & $0.03^{*}$ \\
Cirrhosis & $1(50)$ & $1(50)$ & $1.87(0.12,30.45)$ & 0.6
\end{tabular}


Table 1. Demographic data and univariate analysis of atypical and typical presentations of older adults with infectious diseases at the emergency department (Con)

\begin{tabular}{|c|c|c|c|c|}
\hline Factors & $\begin{array}{l}\text { Atypical } \\
(n=64)\end{array}$ & $\begin{array}{l}\text { Typical } \\
(\mathrm{n}=119)\end{array}$ & $\begin{array}{c}\text { Unadjusted OR } \\
(95 \% \mathrm{CI})\end{array}$ & $\boldsymbol{P}$ \\
\hline Multimorbidity (\%) & $42(38)$ & $69(63)$ & $1.38(0.74,2.6)$ & 0.31 \\
\hline Number of medications (median, IQR 1,3) & $4(0,6)$ & $4(0,7)$ & $1(0.92,1.1)$ & 0.93 \\
\hline \multicolumn{5}{|l|}{ Vital signs } \\
\hline Body temperature ( C) & $37.7(37,38.5)$ & $37.4(36.8,38.2)$ & $1.3(0.97,1.73)$ & 0.08 \\
\hline Pulse rate $>90$ beats $/ \mathrm{min}$ & $36(45)$ & $44(55)$ & $2.13(1.15,3.96)$ & $0.02^{*}$ \\
\hline Respiratory rate (breaths/min) & $20(20,24)$ & $20(20,24)$ & $1.01(0.96,1.06)$ & 0.75 \\
\hline Systolic blood pressure (mmHg) & $132(109.5,146)$ & $133(118,152)$ & $0.99(0.98,1.01)$ & 0.43 \\
\hline Diastolic blood pressure (mmHg) & $75.5(68,84)$ & $73(66,81)$ & $1.02(0.99,1.04)$ & 0.14 \\
\hline
\end{tabular}

OR odds ratio, CI confidence interval, ${ }^{*} P<0.05$, IQR interquartile range, UTI urinary tract infection, Septic shock: suspected or microbiologically proven infection together with systemic inflammatory response syndrome (SIRS) and hypotension [22]. All patients with septic shock from any cause, such as patients with pneumonia and shock, were counted as part of the septic shock group. There were 59 patients in the atypical group with their number of medications available as data, and 119 in the typical group. There were 115 patients in the typical group with available temperature measurements, 117 with pulse rate, respiratory rate, and systolic blood pressure, and 116 with diastolic blood pressure measurements.

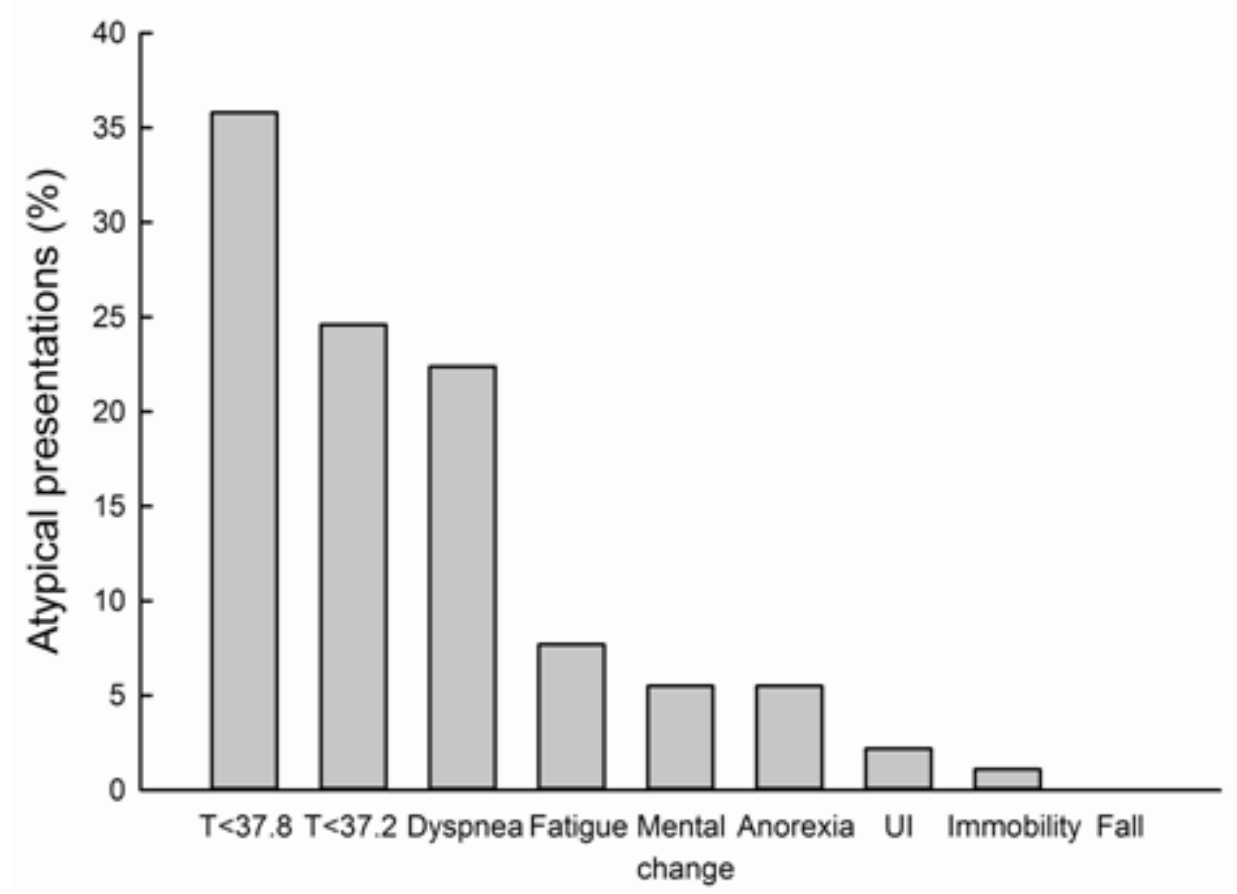

Figure 1. Common atypical presentations of common infectious diseases (\%) in the ED. Common infectious diseases included acute pneumonia or bronchitis, infective diarrhea, complicated urinary tract infection, skin infection, and septic shock. $\mathrm{T}<37.8$, body temperature $<37.8^{\circ} \mathrm{C}, \mathrm{T}<37.2$ body temperature $<37.2^{\circ} \mathrm{C}$, UI urinary incontinence 
Table 2. Multivariate regression model of factors associated with atypical presentations

\begin{tabular}{llllll}
\hline Factors & Unadjusted OR $^{\mathbf{a}}$ & $\mathbf{9 5 \%} \mathbf{C I}^{\mathbf{a}}$ & Adjusted OR & $\mathbf{9 5 \% \mathbf { C I }}$ & $\mathbf{P}$ \\
\hline Age & 1.05 & $(1.0,1.09)$ & 1.01 & $(0.96,1.07)$ & 0.57 \\
$\begin{array}{l}\text { Diagnosis } \\
\quad \text { Complicated UTI }\end{array}$ & 5.56 & $(2.36,13.1)$ & 4.54 & $(1.75,11.78)$ & $0.002^{*}$ \\
$\quad \begin{array}{l}\text { Infective diarrhea } \\
\quad \text { Skin infection }\end{array}$ & 0.2 & $(0.08,0.55)$ & 0.27 & $(0.09,0.8)$ & $0.02^{*}$ \\
$\quad \begin{array}{l}\text { Comorbidity } \\
\quad \text { Cancer }\end{array}$ & 0.1 & $(0.06,0.17)$ & 0.42 & $(0.11,1.64)$ & 0.21 \\
$\quad \begin{array}{l}\text { Dementia } \\
\text { Vital sign }\end{array}$ & 2.5 & $(1.1,5.64)$ & 2.64 & $(1.07,6.53)$ & $0.04^{*}$ \\
$\quad$ Pulse rate >90 beats/min & 2.75 & $(1.18,19.05)$ & 6.66 & $(1.47,30.11)$ & $0.01^{*}$ \\
& & $(1.15,3.96)$ & 2.06 & $(1.01,4.22)$ & $0.04^{*}$ \\
\hline
\end{tabular}

OR odds ratio, CI confidence interval, $P$ was significant at $P<0.05, * P<0.05$, UTI urinary tract infection.

${ }^{a}$ As seen in Table 1.

\section{Discussion}

Infection was a common cause of visits to the ED and accounted for about $29 \%$ of visits by older adults during 2013. The figure is much higher than those reported previously, which were 3\%-14\% [1$3]$. Acute pneumonia or bronchitis was a leading cause of infectious disease, which is consistent with findings from previous studies using data from hospitals throughout Thailand in which respiratory disease was a major cause of hospitalization [6, 21]. Approximately a third (35\%) of older adults with infectious diseases presented atypically.

Common manifestations of atypical presentations of infectious diseases among this age group in previous reports were absence of fever, falls, mental changes, or a decline in general health and nonspecific functional deterioration, which might be the only clinical manifestations of otherwise serious infections $[3,6]$. The results of the present study support findings that older patients with infection have a blunted fever response, even when lowering the cut off from $37.8^{\circ} \mathrm{C}$ to $37.2^{\circ} \mathrm{C}$, which offers greater sensitivity for the detection of fever $[3,5,6]$. Thus, the absence of fever does not exclude serious illness in this population $[5,6]$. Dyspnea is found in about a fifth $(22.4 \%)$ of atypical presentations of common diseases. This symptom did not always originate from a respiratory tract infection. Extrapulmonary causes including septic shock and complicated UTI are also seen. This was apart from cardiac causes, which are the most common presentation of dyspnea in the elderly $[1,22]$.

Although other nonspecific symptoms and signs, including mental changes, fatigue, anorexia, immobility, and urinary incontinence were found, they were fewer in our present study than reported in the literature. Nevertheless, they are still signals of importance. Acute mental change can rapidly lead to delirium and a rate of higher morbidity or mortality [6, 23-25]. Applying rapid, appropriate standardized screening tools such as the Confusion Assessment Method is recommended [26, 27]. Falls as a cause were not found as presenting symptoms in our present study. A possible explanation is the difference in the cultural settings of our Thai population. Most previous studies were conducted in Western countries where older adults usually live alone, and falls tend to occur more often. While in much of Asia, it is still usual that elderly people continue to live within their extended family and have more physical and supervisory support [28]. However, falls should not be neglected, because there is evidence that falls often do not merely represent general frailty, but also underlying pathology that needs to be diagnosed and treated [3, 7]. Fall screening of these patients should be conducted even if they do not report the falls.

Prominent factors associated with atypical presentations of infectious diseases are complicated UTI, cancer, dementia, and pulse rate $>90$ beats $/ \mathrm{min}$. In regard to complicated UTI, generalized nonspecific symptoms predominated over specific symptoms $[9,29]$. It is noteworthy that with higher comorbidities and increased risk of drug-drug and disease-to-disease interactions, the etiologies of UTIs differ greatly between elder and younger adults [9, 30].

Clinicians should consider that in older adults who visit the ED, especially the high-risk group with nonspecific signs and symptoms, urinary retention, high postvoiding residual volume, multiple comorbidities, and patients with short- and long-term 
urinary catheterization history [31, 32], and older patients with cancer are also most likely to have physical impairments because of the side effects of polypharmacy. This group of patients is also likely to show nonspecific presentations [33]. Demented patients have difficulties in communication, cooperation, and physical limitations that can result in an increased risk of atypical presentations and subsequent outcomes. Dementia was associated with UTI in previous studies; partly because of difficulty in managing personal hygiene [2, 34, 35]. Of the vital signs, only high pulse rates $>90$ beats/min was an independent risk factor, and was associated with atypical presentations. A pulse rate of $>90$ beats $/ \mathrm{min}$ increases the risk of early organ failure in older patients with sepsis [11]. Infective diarrhea was protective for developing atypical presentations. Patients with infective diarrhea mostly present with organ specific symptoms and may be specifically diagnosed and treated. Patients with conditions such as an acute abdominal emergency and neurological disorders are usually excluded from this type of study [3, 36]. Advancing age, an absence of fever and tachypnea were not identified as risk factors in previous studies. A possible explanation is that those studies compared the differences between younger and older adults whereas this study focused on elderly adults aged $\geq 65$ years [1, 2, 8, 22].

The present study emphasizes the importance of complete clinical evaluation on presentation. Unexplained tachycardia in the absence of fever and tachypnea did not preclude infectious causes in the elderly patients. Classic signs and symptoms of systemic inflammatory reaction on initial examination may not be evident [3]. Given the high morbidity and mortality of older adults with infection, its recognition may be lifesaving.

Some limitations of this study are recognized. First, the retrospective nature found incompletely recorded vital signs, baseline functional status, and some diseases such as dementia, which might not have been documented on medical records. Second, this study focused on clinical presentations. Known risk factors were not evaluated by laboratory tests. Third, the setting is a tertiary care hospital, where the patients generally have greater disease severity than in a general setting. Finally, this study focused on the ED diagnosis based on an initial assessment. Additional medical problems might be identified in a follow-up period.

\section{Conclusions}

Older adults frequently present with an absence of classic symptoms and signs of infection. Complicated UTI, underlying cancer, and pulse rates $>90$ beats/min were independent risk factors associated with atypical presentations of infection.

\section{Acknowledgments}

We thank Prof. Christopher C. Salisbury and Mr. Dylan Southard, of the Faculty of Medicine Publication Clinic, Khon Kaen University, Thailand for their editing of the manuscript. This study was supported by the Thailand Research Fund (No. IRG 5780016). Publication of this article is subject to an agreement between the authors and Elsevier, who have provided a license (No. 3884940336629) for reproduction of excerpts of text published in Archives of Gerontology and Geriatrics in 2015;62: 97-102.

\section{Conflict of interest statement}

The author(s) declared no potential conflicts of interest with respect to the authorship and/or publication of this article.

\section{References}

1. Chou MY, Chou SL, Tzeng YM, Chen LK, Yen DH, Hwang SJ, et al. Emergency department (ED) utilization and outcome of oldest old men presenting with geriatric syndromes in a veterans care home in Taiwan. Arch Gerontol Geriatr. 2009; 49Suppl 2:S32-6.

2. Salvi F, Morichi V, Grilli A, Giorgi R, De Tommaso G, Dessi-Fulgheri P. The elderly in the emergency department: a critical review of problems and solutions. Intern Emerg Med. 2007; 2:292-301.

3. Samaras N, Chevalley T, Samaras D, Gold G. Older patients in the emergency department: a review. Ann Emerg Med. 2010;56:261-9.

4. Yahav D, Schlesinger A, Daitch V, Akayzen Y, Farbman L, Abu-Ghanem Y, et al. Presentation of infection in older patients—a prospective study. Ann Med. 2015; 47:1-5.

5. Chung MH, Huang CC, Vong SC, Yang TM, Chen KT, Lin HJ, et al. Geriatric fever score: a new decision rule for geriatric care. PLoS One. 2014; 9:e110927.

6. Marco CA, Schoenfeld CN, Hansen KN, Hexter DA, Stearns DA, Kelen GD. Fever in geriatric emergency patients: clinical features associated with serious illness. Ann Emerg Med. 1995; 26:18-24. 
7. Rutschmann OT, Chevalley T, Zumwald C, Luthy C, Vermeulen B, Sarasin FP. Pitfalls in the emergency department triage of frail elderly patients without specific complaints. Swiss Med Wkly. 2005; 135: 145-50.

8. Lee CC, Chen SY, Chang IJ, Chen SC, Wu SC. Comparison of clinical manifestations and outcome of community-acquired bloodstream infections among the oldest old, elderly, and adult patients. Medicine (Baltimore). 2007; 86:138-44.

9. Arinzon Z, Shabat S, Peisakh A, Berner Y. Clinical presentation of urinary tract infection (UTI) differs with aging in women. Arch Gerontol Geriatr. 2012; 55: 145-7.

10. Rehman HU, Qazi S. Atypical manifestations of medical conditions in the elderly. Can Geriatr J. 2013; 3:17-24

11. Wester AL, Dunlop O, Melby KK, Dahle UR, Wyller TB. Age-related differences in symptoms, diagnosis and prognosis of bacteremia. BMC Infect Dis. 2013; 13:346.

12. Limpawattana $\mathrm{P}$, Phungoen $\mathrm{P}$, Mitsungnern $\mathrm{T}$, Laosuangkoon W, Tansangworn N. Atypical presentations of older adults at the emergency department and associated factors. Arch Gerontol Geriatr. 2016; 62:97-102.

13. Ham RJ, Sloane PD, Warshaw GA. Primary care geriatrics: a case based approach. 4th ed. St. Louis: MO: Mosby; 2001.

14. Emmett KR. Nonspecific and atypical presentation of disease in the older patient. Geriatrics. 998; 53:50-2, 8-60.

15. Uijen AA, van de Lisdonk EH. Multimorbidity in primary care: prevalence and trend over the last 20 years. Eur J Gen Pract. 2008; 14Suppl 1:28-32.

16. Longo D, Fauci A, Kasper D, Hauser S, Jameson J, Loscalzo J. Harrison's principles of internal medicine. 18th ed. New York: McGraw Hill; 2012.

17. Barreto ML, Teixeira MG, Carmo EH. Infectious diseases epidemiology. J Epidemiol Community Health. 2006; 60:192-5.

18. Vanpee D, Swine C, Vandenbossche P, Gillet JB. Epidemiological profile of geriatric patients admitted to the emergency department of a university hospital localized in a rural area. Eur J Emerg Med. 2001; 8: 301-4.

19. Chirawatkul A. Biostatistics for medical sciences. Khon Kaen: Klang Na NaWittaya; 2008.

20. Katz MH. Multivariable analysis: a practical guide for clinicians. 2nd ed. Cambridge: Cambridge University
Press; 1999.

21. Limpawattana P, Sutra S, Thavornpitak Y, Wirasorn K, Chindaprasirt J, Mairieng P. Health situation analysis of hospitalized Thai older persons in the year 2010 . J Med Assoc Thai. 2012; 95(Suppl 7):S81-6.

22. Amella EJ. Presentation of illness in older adults. Am J Nurs. 2004; 104:40-51.

23. Lewis LM, Miller DK, Morley JE, Nork MJ, Lasater LC. Unrecognized delirium in ED geriatric patients. Am J Emerg Med. 1995; 13:142-5.

24. Naughton BJ, Moran MB, Kadah H, Heman-Ackah Y, Longano J. Delirium and other cognitive impairment in older adults in an emergency department. Ann Emerg Med. 1995; 25:751-5.

25. Inouye SK, Westendorp RG, Saczynski JS. Delirium in elderly people. Lancet. 2014; 383:911-22.

26. Fong TG, Tulebaev SR, Inouye SK. Delirium in elderly adults: diagnosis, prevention and treatment. Nat Rev Neurol. 2009; 5:210-20.

27. Inouye SK. Delirium in older persons. N Engl J Med. 2006; 354:1157-65.

28. Podhisita C. Thai family and household changes: What we don't know? In: Punpuing S, Sunpuwan S, editors. Thailand's population in transition: a turning point for Thai society. Bangkok: October Press. 2011; 1:23-41.

29. Matthews SJ, Lancaster JW. Urinary tract infections in the elderly population. Am J Geriatr Pharmacother. 2011; 9:286-309.

30. Shortliffe LM, McCue JD. Urinary tract infection at the age extremes: pediatrics and geriatrics. Am J Med. 2002; 113Suppl1A:55S-66S.

31. Rowe TA, Juthani-Mehta M. Urinary tract infection in older adults. Aging Health. 2013; 9.

32. Rowe TA, Juthani-Mehta M. Diagnosis and management of urinary tract infection in older adults. Infect Dis Clin North Am. 2014; 28:75-89.

33. Ershler WB. Cancer: a disease of the elderly. J Support Oncol. 2003; 1:5-10.

34. Eriksson I, Gustafson Y, Fagerstr m L, Olofsson B. Prevalence and factors associated with urinary tract infections (UTIs) in very old women. Arch Gerontol Geriatr. 2010; 50:132-5.

35. Hustey FM, Meldon SW. The prevalence and documentation of impaired mental status in elderly emergency department patients. Ann Emerg Med. 2002; 39:248-53.

36. Iwasaki S, Yamasoba T. Dizziness and imbalance in the elderly: age-related decline in the vestibular system. Aging Dis. 2015; 638-47. 